\title{
Tunneling Spectroscopy for Electronic Bands in Multi-Walled Carbon Nanotubes with Van Der Waals Gap
}

\author{
Dong-Hwan Choi ${ }^{1,2}$, , Seung Mi Lee ${ }^{2}$, Du-Won Jeong ${ }^{1,3}$, Jeong-O Lee ${ }^{3}$, Dong Han Ha ${ }^{2}$, Myung-Ho Bae ${ }^{2,4}, * \mathbb{D}^{\circ}$ \\ and Ju-Jin $\operatorname{Kim}^{1, *}$ \\ 1 Department of Physics, Jeonbuk National University, Jeonju 54896, Korea; \\ cdh1183@skku.edu (D.-H.C.); duwon@krict.re.kr (D.-W.J.) \\ 2 Korea Research Institute of Standards and Science, Daejeon 34113, Korea; \\ seungmi.lee@kriss.re.kr (S.M.L.); dhha@kriss.re.kr (D.H.H.) \\ 3 Korea Research Institute of Chemical Technology, Daejeon 34114, Korea; jolee@krict.re.kr \\ 4 Department of Nano Science, University of Science and Technology, Daejeon 34113, Korea \\ * Correspondence: mhbae@kriss.re.kr (M.-H.B.); jujinkim@chonbuk.ac.kr (J.-J.K.)
}

check for updates

Citation: Choi, D.-H.; Lee, S.M.; Jeong, D.-W.; Lee, J.-O; Ha, D.H.; Bae, M.-H.; Kim, J.-J. Tunneling Spectroscopy for Electronic Bands in Multi-Walled Carbon Nanotubes with Van Der Waals Gap. Molecules 2021, 26, 2128. https://doi.org/ $10.3390 /$ molecules 26082128

Academic Editors: Sung-Hoon Kim and Wan Soo Yun

Received: 6 March 2021

Accepted: 6 April 2021

Published: 7 April 2021

Publisher's Note: MDPI stays neutral with regard to jurisdictional claims in published maps and institutional affiliations.

Copyright: (c) 2021 by the authors. Licensee MDPI, Basel, Switzerland. This article is an open access article distributed under the terms and conditions of the Creative Commons Attribution (CC BY) license (https:// creativecommons.org/licenses/by/ $4.0 /)$.

\begin{abstract}
Various intriguing quantum transport measurements for carbon nanotubes (CNTs) based on their unique electronic band structures have been performed adopting a field-effect transistor (FET), where the contact resistance represents the interaction between the one-dimensional and threedimensional systems. Recently, van der Waals (vdW) gap tunneling spectroscopy for single-walled CNTs with indium-metal contacts was performed adopting an FET device, providing the direct assignment of the subband location in terms of the current-voltage characteristic. Here, we extend the vdW gap tunneling spectroscopy to multi-walled CNTs, which provides transport spectroscopy in a tunneling regime of $\sim 1 \mathrm{eV}$, directly reflecting the electronic density of states. This new quantum transport regime may allow the development of novel quantum devices by selective electron (or hole) injection to specific subbands.
\end{abstract}

Keywords: van-der-Waals gap; tunneling spectroscopy; multi-walled carbon nanotubes; indium

\section{Introduction}

The van der Waals (vdW) contacts between metals and low-dimensional materials do not disturb the physical properties of the low-dimensional materials at the interface [1-3]. For the vdW metal-semiconducting transition metal chalcogenide (TMDC) junctions, the non-perturbed interface allows a tunable Schottky barrier height with various metals having different work functions, following the Schottky-Mott rule [1]. In this case, to achieve vdW contacts between metals and TMDC, metals are mechanically transferred to the TMDC flakes. In particular, it has been revealed that thermally evaporated In metal on TMDCs can also provide the vdW interface with good contact resistance, contrary to other metals evaporated with high evaporation energy, inducing atomic defects in the TMDC layers [2-5]. For single-walled carbon nanotubes (SWCNTs), the In/SWCNT vdW interface functions as a vacuum gap, allowing vdW gap tunneling spectroscopy for the electronic band in SWCNTs [6].

In this work, we extend the vdW gap tunneling spectroscopy to multi-walled CNTs (MWCNTs): two semiconducting MWCNTs and one metallic MWCNT. Low-temperature transport measurements are performed with indium (In)-contacted MWCNTs to reveal multiple conductance peaks and dips that are interpreted as the electronic density of states (DOS) of MWCNTs. This implies that vdW gap tunneling spectroscopy with In electrodes for CNTs provides a feasible way to assign the subbands during operation of a CNT field-effect transistor (FET). 


\section{Results and Discussion}

\section{1. vdW Gap Tunneling Spectroscopy}

Figure 1a presents schematic illustrations of the electronic DOS as a function of the electronic energy in a semiconductor ( $\mathrm{sm}$ )-MWCNT (the middle panel) with source and drain electrodes. Here, SCn (SVn) is the $n$th subband in the conduction (valence) band in the sm-MWCNT, which corresponds to the van Hove singularity in the DOS of CNT. The Fermi level of the MWCNT ( $\left.E_{\mathrm{FC}}\right)$ is located near the conduction band edge (i.e., SC1) in the bandgap. The Fermi levels of the source $\left(E_{\mathrm{FS}}\right)$ and drain $\left(E_{\mathrm{FD}}\right)$ electrodes are also aligned with $E_{\mathrm{FC}}$ under zero bias conditions (the dashed vertical line in the source and drain electrode diagram). The main difference from a conventional field-effect transistor (FET) with Ohmic contacts is the existence of the vdW gap between the source (drain) and MWCNT. In this case, a negative source-drain bias voltage $\left(V_{\mathrm{sd}}\right)$ applied to the source electrode shifts $E_{\mathrm{FS}}$ to a higher energy, causing $E_{\mathrm{FS}}$ to become aligned with SC1, as indicated by the red line in the source electrode diagram. Then, electrons in the source electrode start to tunnel into the MWCNT though the vdW gap, resulting in a current (I) flow, and the differential conductance $\left(\mathrm{d} I / \mathrm{d} V_{\mathrm{sd}}\right)$ at a given $V_{\mathrm{sd}}$ is proportional to the DOS in the MWCNT. By scanning $V_{\mathrm{sd}}$, one can obtain the DOS $(E)$ for the valence and conduction bands.

(a)

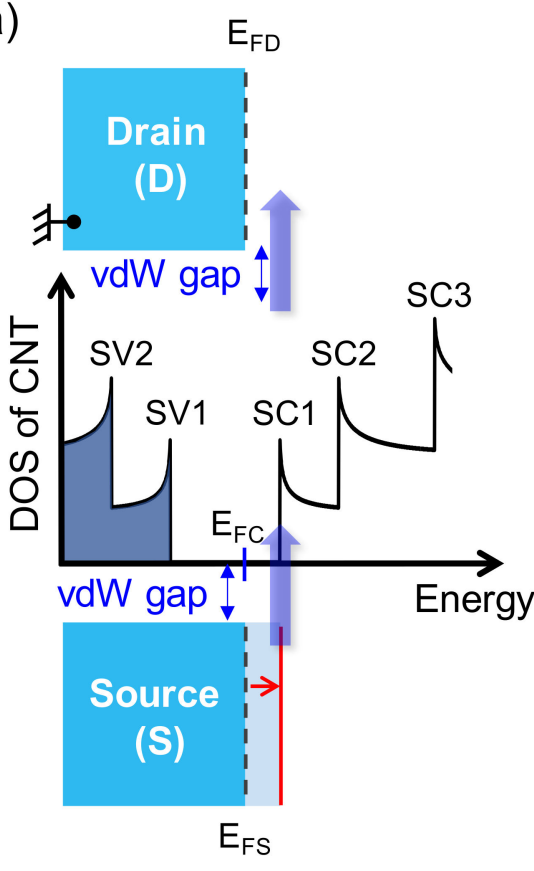

(b)

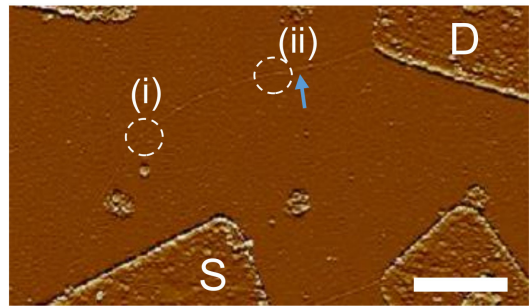

(c)

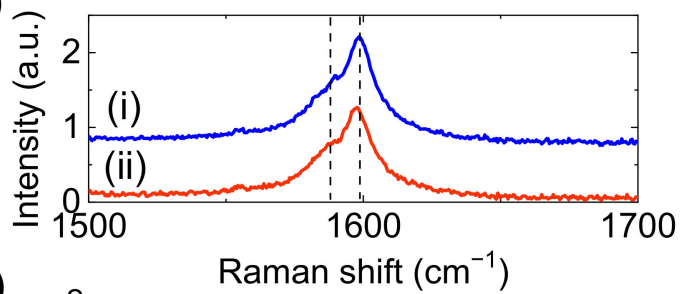

(d)

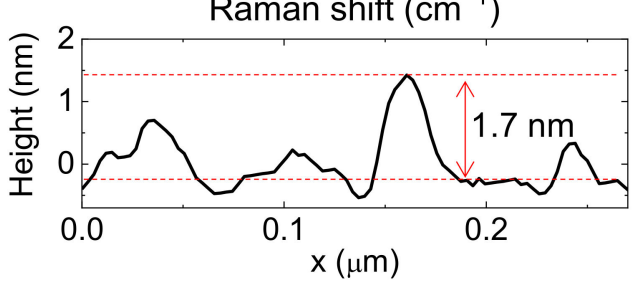

Figure 1. (a) Schematic illustration of the electronic DOS of an MWCNT with two indium electrodes. $E_{\mathrm{FD}}, E_{\mathrm{FS}}$ and $E_{\mathrm{FC}}$ are the Fermi energies of the drain (D), the source (S) and the CNT, respectively. The solid red lines represent $E_{\mathrm{FS}}$ at a finite negative $V_{\mathrm{sd}}$, where $E_{\mathrm{FS}}$ is aligned with $\mathrm{SC} 1$ in the $\mathrm{CNT}$ and results in an enhancement of the conductance, as indicated by the blue arrow. (b) AFM image of a representative $s m$-DWCNT FET device prepared on a $500 \mathrm{~nm}$ thick $\mathrm{SiO}_{2} / \mathrm{Si}$ substrate. The scale bar represents $1 \mu \mathrm{m}$. (c) Raman signals obtained from circled regions (indicated by (i) and (ii) in (b)), which show a main peak at $1598 \mathrm{~cm}^{-1}$ as a $G$ peak with a satellite peak at $1586 \mathrm{~cm}^{-1}$. (d) Height profile along the direction of the blue arrow in (b).

\subsection{Characterization of DWCNT}

To realize the vdW gap between the metal and MWCNT, we deposit $60 \mathrm{~nm}$ thick In metal for the source and drain electrodes on a double-walled CNT (DWCNT) located on a $500 \mathrm{~nm}$ thick $\mathrm{SiO}_{2} / \mathrm{Si}$ substrate with a back-gate geometry, as shown in Figure $1 \mathrm{~b}$. During the deposition, the substrate stage in the vacuum chamber was kept at $100 \mathrm{~K}$ with liquid nitrogen to obtain homogeneity of the In film [6]. The DWCNT channel length $(L)$ 
was $\sim 6 \mu \mathrm{m}$. The homogeneity of the DWCNT was checked by the Raman spectra at two points $\sim 2 \mu \mathrm{m}$ apart. For instance, Figure 1c shows the Raman spectra of (i) and (ii) obtained from the dashed circled regions indicated by (i) and (ii) in Figure 1b, respectively, with a $514 \mathrm{~nm}$ wavelength laser. Both spectra show a main $\mathrm{G}$ peak at $1598 \mathrm{~cm}^{-1}$ with a satellite peak at $1586 \mathrm{~cm}^{-1}$ (see two vertical dashed lines). This is consistent with a previous report presenting DWCNTs with a similar laser wavelength [7]. Figure 1d shows the height profile obtained from the AFM scanning along the direction of the arrow in Figure 1b, which provides the diameter $(D)$ of the DWCNT, $\sim 1.7 \mathrm{~nm}$.

\section{3. vdW Tunneling Spectroscopy for DWCNT}

Figure 2a shows the transfer curve, current $(I)$ as a function of back-gate voltage $\left(V_{\mathrm{bg}}\right)$ at source-drain voltage $\left(V_{\mathrm{sd}}\right)$ of $2 \mathrm{~V}$ for the DWCNT, which exhibits semiconducting (sm) behavior at $T=10 \mathrm{~K}$. The $\mathrm{d} I / \mathrm{d} V_{\text {sd }}$ map for the $s m$-DWCNT in Figure $2 \mathrm{~b}$ shows that the DWCNT is slightly intrinsically hole $(h)$-doped. $V_{\mathrm{sd}}$, corresponding to the multiple conductance peaks observed for $V_{\mathrm{bg}}<-20 \mathrm{~V}$ in Figure $2 \mathrm{~b}$, seems to be insensitive to $V_{\mathrm{bg}}$. However, near $V_{\mathrm{bg}}=-15 \mathrm{~V}$, the lines corresponding to the peaks abruptly curve to lie parallel to the depletion boundary that is indicated by the left-hand white dashed line in the yellow oval. In Figure 2c, when $V_{\mathrm{bg}}$ decreases across $V_{\mathrm{bg}}=-20 \mathrm{~V}$, an additional conductance peak appears at $V_{\mathrm{sd}}=0.32 \mathrm{~V}$, as indicated by the dotted circle at $V_{\mathrm{bg}}=-25 \mathrm{~V}$. This corresponds to the newly appeared conductance peak curve for $V_{\mathrm{bg}}<-20 \mathrm{~V}$, as indicated by an arrow in Figure $2 \mathrm{~b}$. This implies that a subband with a relatively lower index number can be detected at a sufficiently high negative $V_{\mathrm{bg}}$ region. This is because a relatively large bandgap causes noticeable charge depletion in the CNT near the metal interface with the corresponding bias conditions $\left(V_{\mathrm{bg}} \sim-20 \mathrm{~V}\right)$, which suppresses the tunnel probability. To understand this, we construct a band model, as described in the following section.

(a)

(b)
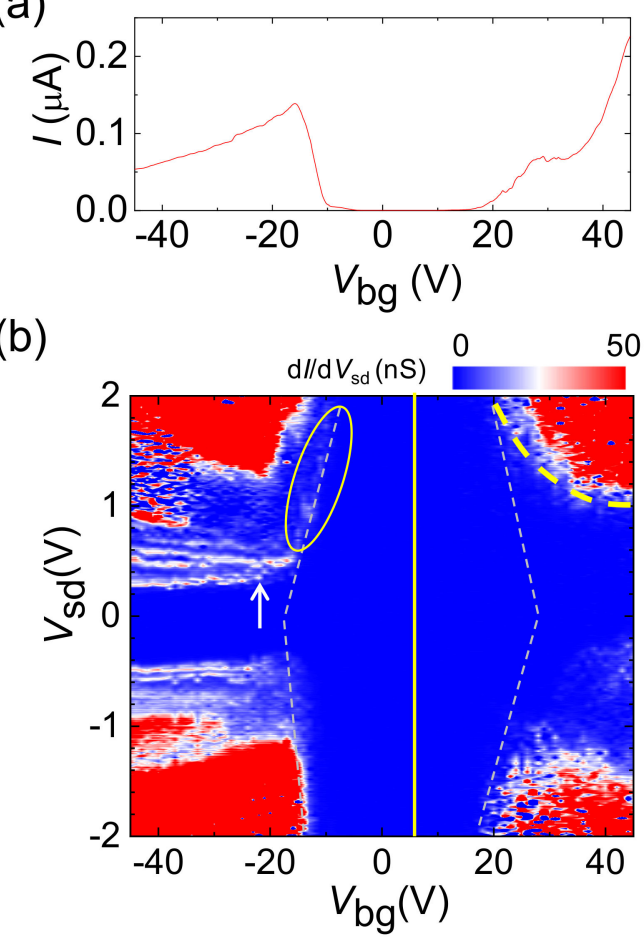

(c)

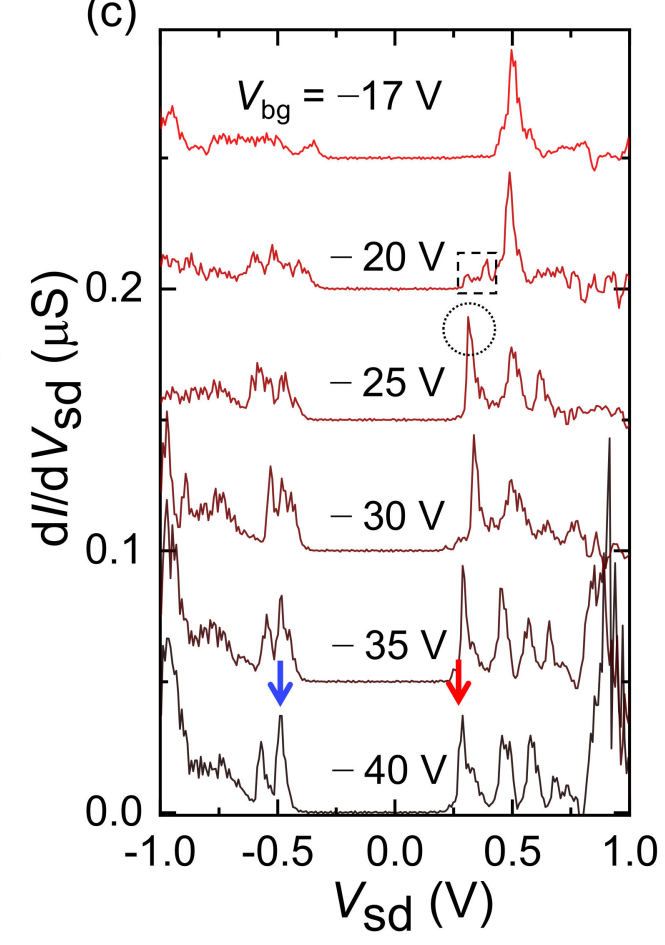

Figure 2. (a) $I-V_{\mathrm{bg}}$ (transfer) curve for an $\operatorname{sm}$-DWCNT $(L \approx 6 \mu \mathrm{m}, D \approx 1.7 \mathrm{~nm})$ at $V_{\mathrm{sd}}=2 \mathrm{~V}$ and $T=10 \mathrm{~K}$. (b) $\mathrm{d} I / \mathrm{d} V_{\text {sd }}$ map as a function of $V_{\text {sd }}$ and $V_{\text {bg }}$ of the sm-DWCT, where the dashed lines indicate the boundaries of the depletion region. The vertical line indicates the midgap location, $E_{\mathrm{g}} / 2$. (c) $\mathrm{d} I / \mathrm{d} V_{\text {sd }}$ as a function of $V_{\text {sd }}$ for various $V_{\mathrm{bg}}$ of the $s m$-DWCNT. 


\subsection{Band Model for sm-DWCNT Tunnel Junction}

The slightly $h$-doped state indicates that the $E_{\mathrm{F}}$ of the source (S) and drain (D) electrodes are located at a lower location from the mid-gap $\left(E_{\mathrm{g}} / 2\right)$ of the $s m$-CNT (see the vertical line in Figure $2 b$ ). In the gap region, Figure $2 b$ shows zero conductance for examined $V_{\text {sd }}$ up to $\pm 2 \mathrm{~V}$ at $-10 \mathrm{~V}<V_{\mathrm{bg}}<20 \mathrm{~V}$. Near $V_{\mathrm{bg}} \sim 7 \mathrm{~V}$, the bands are nearly flat (see the left panel of Figure 3a). With positively increasing $V_{\mathrm{bg}}$, the bands become pulled towards a lower direction while the ends of the CNT bands keep their original positions as shown by the green curves in the middle panel of Figure 3a [8], where only the lowest (SC1) and highest (SV1) bands of the conduction and valance bands are depicted, respectively. In this case, although a positive $V_{\mathrm{sd}}$ comparable to $E_{\mathrm{g}}$ is applied, currents cannot flow through the CNT due to the thick potential barrier indicated by the dashed green circle for the green band in the right-hand panel of Figure $3 \mathrm{a}$. For $V_{\mathrm{bg}}>20 \mathrm{~V}$ in Figure $2 \mathrm{~b}$, the bands in the $\mathrm{CNT}$ are significantly bent, as shown by the red curves in the middle of Figure 3a. At the same $V_{\text {sd }}$ condition as the green bands, electrons then encounter a relatively thin potential barrier, as indicated by the red dashed circle for the red bands in the right-hand panel of Figure 3a. This allows the current to flow through the DWCNT and forms the conductance boundary indicated by the dashed yellow curve in Figure $2 b$.

(a) vdW tunnel barrier

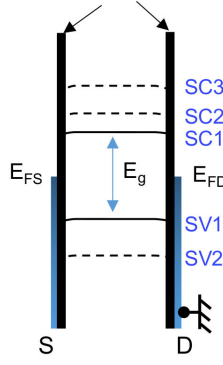

(b)

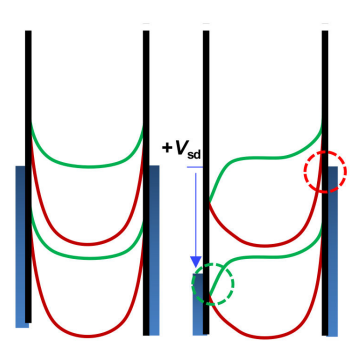

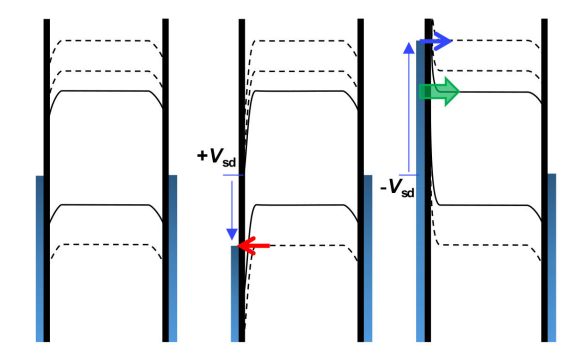

(c)

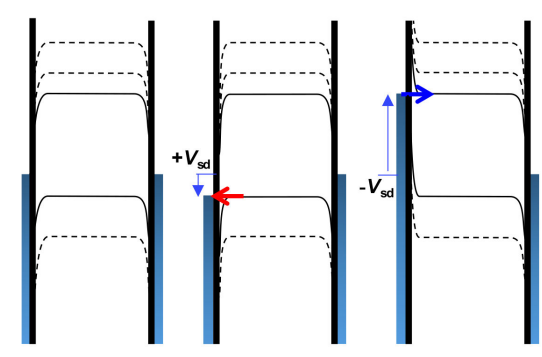

Figure 3. (a) Left: electronics bands for $V_{\mathrm{bg}}=7 \mathrm{~V}$, middle and right: electronic bands for $7 \mathrm{~V}<V_{\mathrm{bg}}<20 \mathrm{~V}$ (green curves) and $V_{\mathrm{bg}}>20 \mathrm{~V}$ (red curves). SCn (SVn) is the $n$th subband in the conduction (valence) band. Electronic bands for (b) $-20 \mathrm{~V}$ $<V_{\mathrm{bg}}<-17 \mathrm{~V}$ and $(\mathrm{c}) V_{\mathrm{bg}}<-22 \mathrm{~V}$.

In a hole-doped region for $V_{\mathrm{bg}}<7 \mathrm{~V}$, the DWCNT bands are pulled towards an upper direction. For $V_{\mathrm{bg}} \sim-10 \mathrm{~V}$ in Figure $2 \mathrm{~b}$, the maximum energy of the valence band is still lower than the $E_{\mathrm{F}}$ of the electrodes, as shown in the left-hand panel of Figure $3 \mathrm{~b}$. With positively increasing $V_{\mathrm{sd}}$ in the middle panel of Figure $3 \mathrm{~b}$, the potential barrier width at the source becomes narrower and a tunnel event occurs. The middle of Figure $3 \mathrm{~b}$ shows a case of detectable tunneling current when $E_{\mathrm{FS}}$ is entered into a certain subband with a sufficiently positive $V_{\text {sd }}$ (see the red arrow in the middle panel of Figure $3 \mathrm{~b}$ ), which will produce a conductance peak, as observed at $V_{\mathrm{sd}} \sim 0.5 \mathrm{~V}$ for $V_{\mathrm{bg}}=-17$ and $-20 \mathrm{~V}$ in Figure 2c. We found an irregular conductance region in the dashed box at $V_{\mathrm{bg}}=-20 \mathrm{~V}$ in Figure 2c, located at a lower $V_{\text {sd }}$ than that of the main peak at $V_{\text {sd }} \sim 0.5 \mathrm{~V}$. This could be related to a lower subband. For instance, the tunneling probability through the low-index subband with a relatively low $V_{\text {sd }}$ value would be too low to measure a pronounced conductance peak, due to the relatively thick potential barrier. If a negative $V_{\text {sd }}$ is applied near $V_{\text {bg }} \sim-20 \mathrm{~V}$, the Zener tunneling current (green arrow) with the general tunneling current (blue arrow) becomes involved, as shown in the right-hand panel of Figure $3 b$, resulting in the smearing of local conductance peaks for negative $V_{\text {sd }}$.

With more negative $V_{\mathrm{bg}}$ in Figure $2 \mathrm{~b}$, the bands are more bent and the maximum of the valance band more approaches the Fermi levels of the electrodes, as depicted in the left-hand panel of Figure 3c. In this case, it could provide a detectable tunneling current when $E_{\mathrm{FS}}$ is aligned to the low subband with a positive $V_{\mathrm{sd}}$. This change is indeed shown in Figure $2 \mathrm{~b}$, indicated by the vertical arrow at $V_{\mathrm{bg}}=-22 \mathrm{~V}$, where a new conductance peak curve is introduced with negatively increasing $V_{\mathrm{bg}}$. At $V_{\mathrm{sd}}=-25 \mathrm{~V}$ in Figure 2c, 
the new peak appears in the irregular conductance region, thus we consider that the new peak should have a lower subband index number than that for the peak at $V_{\mathrm{bg}}=-20 \mathrm{~V}$. For a negative $V_{\mathrm{sd}}$, the tunneling current through the subbands in the conduction band is also expected before entering into the Zener regime because the tunnel barrier is already narrowed, as shown in the right-hand panel of Figure 3c. With negatively increasing $V_{\mathrm{bg}}$, the new peak follows the conductance curve indicated by the arrow in Figure $2 \mathrm{~b}$ and corresponds to the peak indicated by the red arrow at $V_{\mathrm{sd}} \sim 0.25 \mathrm{~V}$ and $V_{\mathrm{bg}}=-40 \mathrm{~V}$ in Figure 2c. Since the bands become bent towards the upper direction with negatively increasing $V_{\mathrm{bg}}$, the tunnel probability becomes higher for negatively increasing $V_{\mathrm{bg}}$. Then, there should be finite conductance for $V_{\mathrm{sd}}<0.25 \mathrm{~V}$ if there are low-index subbands, as in the irregular conductance region in the dashed box at $V_{\mathrm{bg}}=-20 \mathrm{~V}$. However, we could not identify a noticeable finite conductance in the $V_{\text {sd }}$ region, which suggests that the peak indicated by the red arrow corresponds to the lowest subband, SV1. Then, the peak at $V_{\mathrm{sd}} \sim 0.5 \mathrm{~V}$ for $V_{\mathrm{bg}}=-20 \mathrm{~V}$ can be considered as SV2. Following a similar analysis, it is suggested that the peak indicated by the blue arrow at $V_{\mathrm{bg}}=-40 \mathrm{~V}$ corresponds to SC1.

\subsection{Density Functional Theory (DFT) Calculations for DWCNTs}

We consider that the tunneling between the electrode and DWCNT occurs based on the hybridized electronic band structure between inner and outer CNTs [9-11]. In order to theoretically determine the electronic structure of DWCNTs, we performed DFT calculations with five different SWCNTs, the components of DWCNTs, as listed in Table 1. Although the DFT method has been known to underestimate the electronic gap when using a "conventional" exchange-correlation functional of local density approximation (LDA) or GGA functional, the comparative study of the $E_{\mathrm{g}}$ of similar structures, such as CNTs, is still considered practical and relevant. Table 1 shows the chiralities, diameters, the calculated $E_{\mathrm{g}}$ and the electrical properties of SWCNTs. We selected the potential outer tube with a diameter of $1.3 \sim 1.5 \mathrm{~nm}$ and then selected the relevant inner tube. Only zigzag tubes were considered due to their various electronic properties according to the chirality and also the relatively short repeating unit length in calculational geometry along the tube axis. The calculated $E_{\mathrm{g}}$ values show the expected well-known trends, which are semiconducting for $(3 n+1,0)$ or $(3 n+2,0)$ and metallic for $(3 n, 0)$ SWCNTs. The small bandgap for $(9,0)$ originated from the large $\pi^{*}-\sigma^{*}$ hybridization in CNTs with a small diameter, as reported previously [12]. This $\pi^{*}-\sigma^{*}$ hybridization effect becomes negligible as the diameter increases, as we can see clearly when comparing the $E_{\mathrm{g}}$ of $(9,0)$ and $(18,0)$ SWCNTs listed in Table 1. In order to interpret our experimental observations, we calculated the electronic properties of DWCNTs. Using the SWCNTs listed in Table 1, we generated four DWCNTs with properties of $s m @ s m, m @ s m$, sm@m and $m @ m$ (inner@outer CNTs), keeping the intertube distance close to the van-der-Waals distance, where $m$ indicates a metal. The calculated electronic bandgap and the properties are listed in Figure 4 and Table 2. For the sm@sm case, $E_{\mathrm{g}}$ did not change significantly from the original value. However, in the case of $m @ s m$, $E_{\mathrm{g}}$ was suppressed by as much as a factor of two, although it retained the semiconducting property, which was due to the overlapping of the electrons' wavefunctions in the metallic and semiconducting CNTs. On the other hand, both $s m @ m$ and $m @ m$ showed metallic behaviors while retaining the electronic structure of the inner CNT. A recent systematic study showed that the $E_{\mathrm{g}}$ of DWCNTs is smaller than the average value of the $E_{\mathrm{g}} \mathrm{s}$ of inner and outer tubes, showing the metallization trend of DWCNT [13]. Our experimental results for the DWCNT in Figure 2 support the case of sm@sm in Figure 4a, with a considerable $E_{\mathrm{g}}$ of $\sim 0.7 \mathrm{eV}$. 
Table 1. Chiralities, diameters, the calculated electronic bandgap $\left(E_{\mathrm{g}}\right)$ and the electrical properties of selected SWCNTs.

\begin{tabular}{cccccc}
\hline Chirality & $\mathbf{( 8 , 0 )}$ & $\mathbf{( 9 , 0 )}$ & $\mathbf{( 1 0 , 0 )}$ & $\mathbf{( 1 7 , 0 )}$ & $\mathbf{( 1 8 , 0 )}$ \\
\hline Diameter $(\AA)$ & 6.26 & 7.05 & 7.83 & 13.32 & 14.10 \\
$E_{\mathrm{g}}(\mathrm{eV})$ & 0.55 & 0.10 & 0.91 & 0.54 & 0.02 \\
Property & $s m$ & $(m)$ & $s m$ & $s m$ & $(m)$ \\
\hline
\end{tabular}

Table 2. Chiralities in the inner@outer CNT format, component properties, the calculated electronic $E_{\mathrm{g}}$ and the electrical properties of DWCNTs composed with SWCNTs listed in Table 1.

\begin{tabular}{ccccc}
\hline Chirality & $\mathbf{( 8 , 0 ) @ ( \mathbf { 1 7 } , \mathbf { 0 } )}$ & $\mathbf{( 9 , 0 ) @ ( \mathbf { 1 7 } , \mathbf { 0 } )}$ & $\mathbf{( 1 0 , 0 ) @ ( 1 8 , 0 )}$ & $\mathbf{( 9 , 0 ) @ ( 1 8 , 0 )}$ \\
\hline Component Property & $s m @ s m$ & $m @ s m$ & $s m @ m$ & $m @ m$ \\
$E_{\mathrm{g}}(\mathrm{eV})$ & 0.47 & 0.22 & 0.03 & 0.02 \\
DWCNT property & $s m$ & $s m$ & $(m)$ & $(m)$ \\
\hline
\end{tabular}

(a)

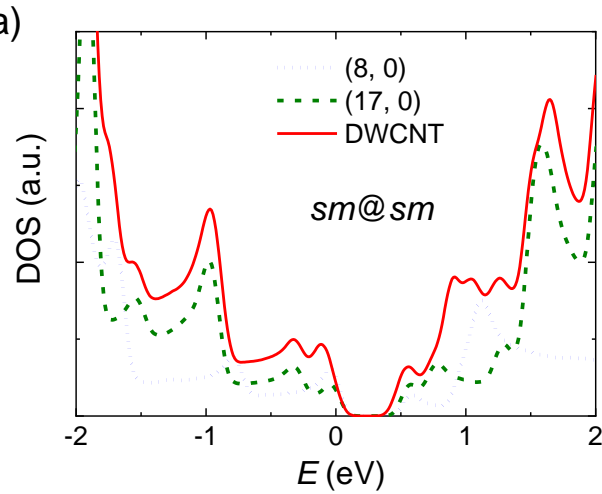

(c)

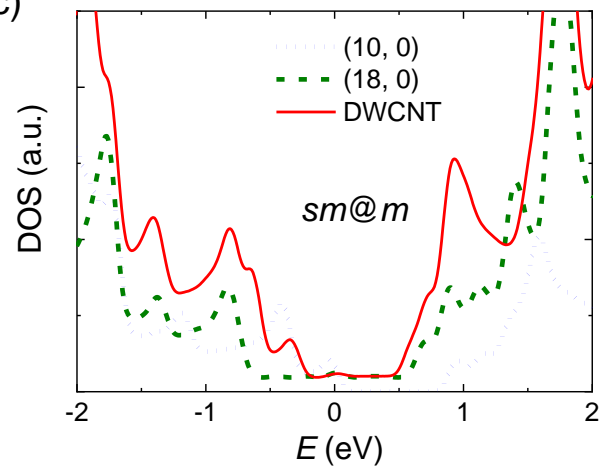

(b)

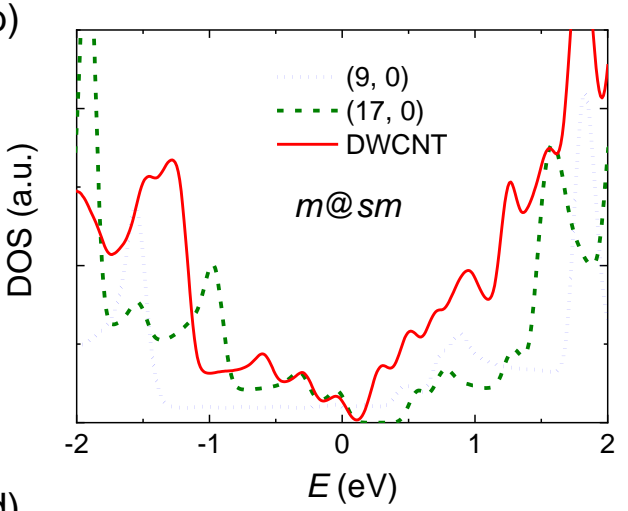

(d)

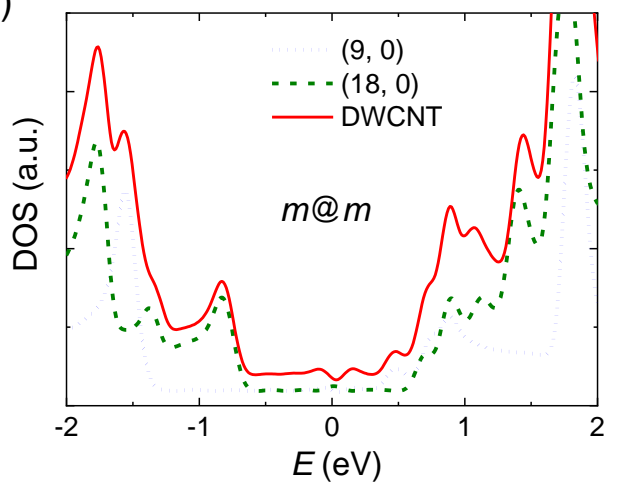

Figure 4. Electronic DOS of DWCNTs, sm@sm (a), $m @ s m(\mathbf{b}), s m @ m(\mathbf{c})$ and $m @ m(\mathbf{d})$, which also show the DOS of SWCNTs constructing DWCNTs. Gaussian broadening with $0.05 \mathrm{eV}$ width has been applied to the eigenvalues obtained from DFT calculations.

\section{6. vdW Tunneling Spectroscopy for MWCNTs}

Figure $5 \mathrm{a}$ shows the transfer curves for various $V_{\text {sd }}$ at $T=4.2 \mathrm{~K}$ for an $s m-M W C N T$ with $D \sim 3 \mathrm{~nm}$ and $L \sim 2.1 \mu \mathrm{m}$ (see Supplementary Figures S1 and S2 for the AFM and Raman characterizations, respectively); this figure reveals $s m$ behavior with an electron (e)-doped ambipolar property. In the $e$-doped region $\left(V_{\mathrm{bg}}>-10 \mathrm{~V}\right)$, the curve shows two current steps at $V_{\mathrm{sd}}=0.75 \mathrm{~V}$, as indicated by two arrows. With decreasing $V_{\mathrm{sd}}$, the steps disappear when the saturation current observed for $V_{\mathrm{bg}}>-5 \mathrm{~V}$ is lower than those of the current steps. The hole $(h)$-doped region $\left(V_{\mathrm{bg}}<-20 \mathrm{~V}\right)$ shows rather complicated current modulation behavior. Figure $5 \mathrm{~b}$ presents a $\mathrm{d} I / \mathrm{d} V_{\text {sd }}$ map as a function of $V_{\mathrm{sd}}$ and $V_{\text {bg }}$. This plot shows several distinct conductance peak curves in both doped regions, as indicated by dashed lines. In the $h$-doped region $\left(V_{\mathrm{bg}}<-20 \mathrm{~V}\right)$, the $V_{\mathrm{sd}}$ for a conductance 
peak curve shows a strong gate dependence, which results in the complicated current modulations observed in this region in Figure $5 \mathrm{~b}$. By contrast, the $V_{\text {sd }}$ for a conductance peak curve in the $e$-doped region is not sensitive to $V_{\mathrm{bg}}$, except near the depletion region, thereby giving rise to the step-like transfer curve with saturation current observed in the $e$-doped region in Figure 5a. Considering the extension of the energy scale to $\sim 1 \mathrm{eV}$ and the distinctive gate dependence of the conductance peaks, their origin does not lie in the formation of a quantum dot or conductance quantization, as has been suggested previously [14,15]. The white dash-dotted lines in Figure 5b represent the boundaries of the depletion region related to the bandgap of the sm-MWCNT. We observed that all of the conductance peak curves bent to lie parallel to the boundaries of the depletion region, which indicates that the conductance peaks could be linked to the DOS related to the electronic band structure of the sm-MWCNT, i.e., van Hove singularities in the subband structures. The blue curve in Figure $5 \mathrm{c}$ displays $\mathrm{d} I / \mathrm{d} V_{\mathrm{sd}}$ as a function of $V_{\mathrm{sd}}$ at $V_{\mathrm{bg}}=0 \mathrm{~V}$; this plot shows six main conductance peaks, as indicated by inverse triangles. We observed the minimum conductance at $V_{\mathrm{sd}}=0.1 \mathrm{~V}$, as indicated by the blue arrow in Figure $5 \mathrm{c}$. If we assume that this minimum corresponds to the mid-gap $\left(E_{g} / 2\right)$ position, then we can assign two conductance peaks at $V_{\text {sd }}=35$ and $155 \mathrm{mV}$, which are the peaks nearest to the mid-gap, labeled SC1 and SV1. With this peak assignment, the Fermi level of the CNT $\left(E_{\mathrm{FC}}\right)$ is located between SC2 and SC1 at $V_{\mathrm{bg}}=V_{\mathrm{sd}}=0 \mathrm{~V}$, as depicted by the vertical dashed line in Figure 5c. The red curve with multiple conductance peaks in Figure $5 \mathrm{c}$ was obtained at $V_{\mathrm{bg}}=-40 \mathrm{~V}$, where the minimum conductance was observed at $V_{\mathrm{sd}} \sim 30 \mathrm{mV}$. The shift in the midgap position in $V_{\text {sd }}$ with different $V_{\text {bg }}$ values occurred because the electronic bands were bent by the varying $V_{\mathrm{bg}}$ values (see Supplementary Materials Sections 1-3). When the red curve was shifted to match the minimum conductance to that observed from the blue curve (see Supplementary Figure S3c), the locations of the main conductance peaks in the two curves are nearly coincident, which confirms that the origins of the multiple conductance peaks are the intrinsic electronic states existing in the sm-MWCNT, van Hove singularities (also see the band model for sm-MWCNT in Supplementary Materials Sections 1-3).

(a)

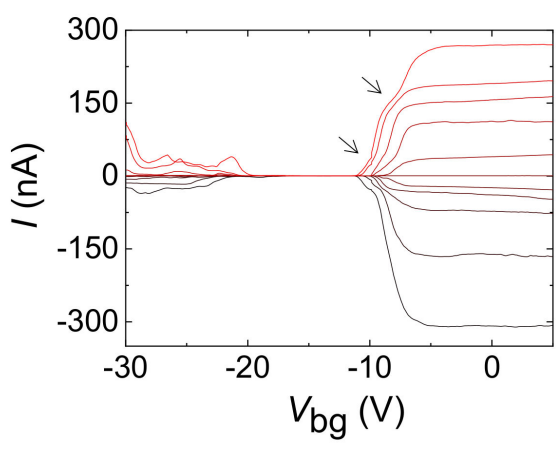

(b)

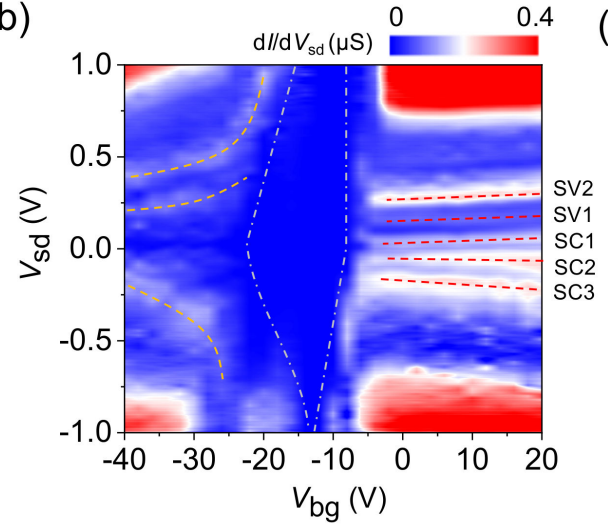

(c)

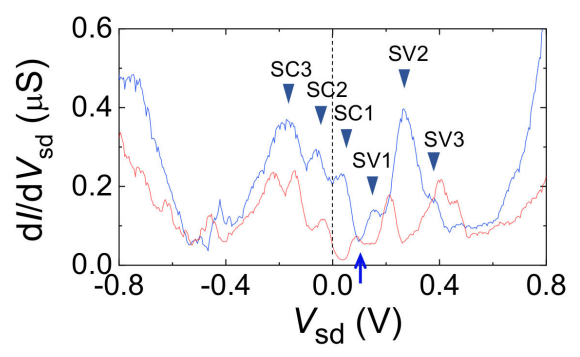

Figure 5. (a) Transfer curve for an $\operatorname{sm}$-MWCNT $(L \approx 2.1 \mu \mathrm{m}, D \approx 3 \mathrm{~nm})$ at $T=4.2 \mathrm{~K}$ for various $V_{\text {sd }}$ values from $0.75 \mathrm{~V}$ (top) to $-0.75 \mathrm{~V}$ (bottom) in steps of $0.15 \mathrm{~V}$. Arrows indicate the current steps, originated from the DOS-sensitive transport.

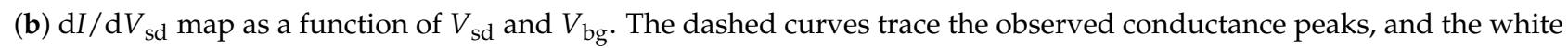
dash-dotted lines correspond to the boundaries of the depletion region. (c) $\mathrm{d} I / \mathrm{d} V_{\text {sd }}$ as a function of $V_{\text {sd }}$ at $V_{\text {bg }}=0 \mathrm{~V}$ (blue curve) and $-40 \mathrm{~V}$ (red curve), where $\mathrm{SC} n(\mathrm{SV} n)$ is the $n$th subband in the conduction (valence) band.

We further examined an $m$-MWCNT with $D(L) \sim 4.8 \mathrm{~nm}(\sim 1 \mu \mathrm{m})$ (see the AFM and Raman characterizations in Supplementary Figure S4). Figure 6a shows the transfer curve, which exhibits a quasi-metallic behavior. The $\mathrm{d} I / \mathrm{d} V_{\text {sd }}$ map as a function of $V_{\mathrm{sd}}$ and $V_{\mathrm{bg}}$ in the left-hand panel of Figure $6 \mathrm{~b}$ exhibits multiple pronounced conductance peaks, as indicated by three arrows, which are indicated by the same color-coded arrows, respectively, at $V_{\mathrm{bg}}=6.2 \mathrm{~V}$ in Figure 6c. We noticed a relatively small peak at $V_{\mathrm{sd}} \sim 70 \mathrm{mV}$ for a curve at $V_{\mathrm{bg}}=6.2 \mathrm{~V}$, as indicated by the blue arrow. After changing the color contrast 
of the left-hand panel of Figure $6 \mathrm{~b}$, we found that the small peak also formed a conductance curve, as indicated by the blue arrow in the right-hand panel of Figure $6 \mathrm{~b}$. There were also several weak but finite conductance curves. Our DFT calculations for the metallic DWCNTs in Figure 4c,d show that it is not feasible to assign the peaks even in the calculation results. With this reason, we could not assign the peaks for the $m$-MWCNT in Figure 6 . For the $m$-MWCNT case, there should be a finite DOS between the lowest subbands, without a bandgap, which manifests as the finite conductance observed at $-0.3 \mathrm{~V}<V_{\mathrm{sd}}<0.3 \mathrm{~V}$ in Figure 6c.

(a)

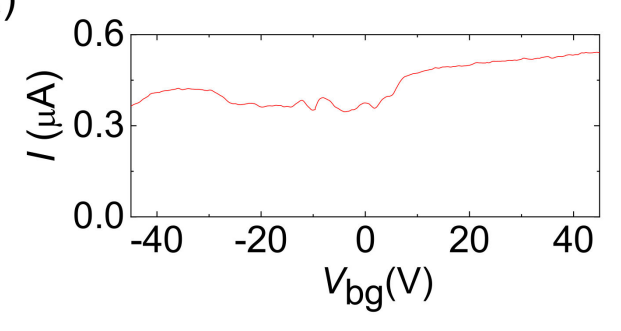

(c)
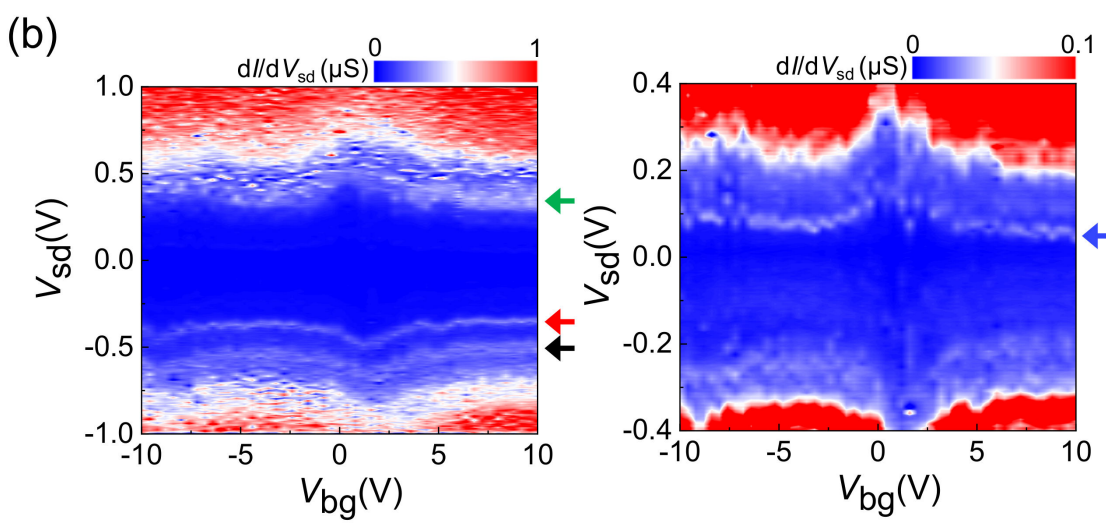

Figure 6. (a) Transfer curve for an $m-\operatorname{MWCNT}(\mathrm{L} \approx 1 \mu \mathrm{m}, \mathrm{D} \approx 4.8 \mathrm{~nm})$ at $V_{s d}=1 \mathrm{~V}$ and $T=4.2 \mathrm{~K}$. (b) Left panel: $\mathrm{d} I / \mathrm{d} V_{\mathrm{sd}}$ as a function of $V_{\mathrm{sd}}$ and $V_{\mathrm{bg}}$ for the $m$-MWCNT. Right panel: Zoomed plot of the left panel with a different contrast. (c) $\mathrm{d} I / \mathrm{d} V_{\mathrm{sd}}$ as a function of $V_{\mathrm{sd}}$ for various $V_{\mathrm{bg}}$ of the $m$-MWCNT.

\section{Materials and Methods}

\subsection{Growth of MWCNT}

A catalyst liquid composed of $\mathrm{CH}_{3} \mathrm{OH}:\left(\mathrm{Fe}\left(\mathrm{NO}_{3}\right)_{3}\right)_{9} \mathrm{H}_{2} \mathrm{O}: \mathrm{Al}_{2} \mathrm{O}_{3}: \mathrm{MoO} 2=15: 20: 15: 5$ (mg) was dropped onto a $500 \mathrm{~nm}$ thick $\mathrm{SiO}_{2} / \mathrm{Si}$ substrate with patterned positioning markers and the liquid was blown by nitrogen gas. MWCNTs were grown in a chemical vapor deposition (CVD) chamber with a gas mixture, $\mathrm{CH}_{4}: \mathrm{H}_{2}=5000: 500(\mathrm{sccm})$, at $915{ }^{\circ} \mathrm{C}$ for $10 \mathrm{~min}$.

\subsection{Theoretical Calculations}

We used density functional theory (DFT) methods. All electrons' Kohn-Sham wave functions were expanded in local atomic orbital basis sets, as implemented in DMOL3 code [16]. The double numeric basis sets with polarization were used and damped atompairwise dispersion corrections with the form $\mathrm{C}_{6} \mathrm{R}^{-6}$ using the Tatchenco-Scheffler method were considered [17]. The $k$-points sampled with equidistance of $0.05 / \AA$ were used for all calculations to maintain the same criterion among CNTs. We used the generalized gradient approximation (GGA) functional proposed by Perdew-Becke-Ernzerhof (PBE) [18]. 


\subsection{Raman and AFM Characterization}

The LabRAM HR system (Horiba Scientific) was used for the Raman spectra with a $514 \mathrm{~nm}$ wavelength laser and a $100 \times$ objective lens (beam exposure time: 10-15 s). The AFM image under tapping mode was obtained with the Park Systems XE-100 AFM.

\subsection{Experiments}

The electrical measurements were performed with a cryo-free system (base temperature: $4 \mathrm{~K}$ ) in two-probe mode with a voltage source (Keithely 213) and current amplifier (DL 1211). The differential conductance as a function of bias voltage was numerically obtained from the current-voltage characteristics.

\section{Conclusions}

In summary, we performed vdW tunneling spectroscopy for MWCNTs with In metal contacts; sm-DWCNT, sm- and $m$-MWCNTs, which revealed the electronic DOS of MWCNTs as shown in conventional scanning tunneling spectroscopy $[19,20]$. The In/MWCNT contact forms a vdW interface, i.e., the electronic DOS of an MWCNT is conserved at the interface, with a vdW vacuum gap. We believe that this is why it is feasible for CNT devices with In metal contacts to provide consistent tunneling spectroscopy. A recent work successfully demonstrated the application of tunneling spectroscopy in SWCNT/hexagonal boron nitride(hBN)/SWCNT heterojunctions, where conductance peaks observed at different $V_{\text {sd }}$ were interpreted as elastic tunneling into the van Hove singularities of different one-dimensional subbands in both SWCNTs through the hBN tunneling barrier [21]. The transport regime reflecting the van Hove singularities could allow the development of new quantum devices operated by controlled electron (or hole) injection to a specific subband in CNT-based FETs.

Supplementary Materials: Supplementary materials are available online. Figure S1: AFM images of $s m$-MWCNT, Figure S2: Raman shift of $s m$-MWCNT, Figure S3: Band model of $s m$-MWCNT, Figure S4: AFM images of $m$-MWCNT.

Author Contributions: Conceptualization, M.-H.B. and J.-J.K.; investigation: D.-H.C., S.M.L., D.-W.J., J.-O.L, D.H.H.; Writing—Original Draft Preparation, M.-H.B., S.M.L. and J.-J.K.; Writing—Review and Editing, all authors. All authors have read and agreed to the published version of the manuscript.

Funding: This research was supported by "Research Base Construction Fund Support Program" funded by Jeonbuk National University in 2020. This work was also partly supported by the Korea Research Institute of Standards and Science (KRISS-2021-GP2021-0001), the National Research Foundation of Korea (NRF) (Grant Nos. SRC2016R1A5A1008184, 2018R1A2A1A05078440, 2021R1A2C3012612) and the National Research Council of Science \& Technology (NST) grant by the Korean Government (MSIT) (No. CAP-18-04-KRISS).

Data Availability Statement: The data presented in this study are available on request from the corresponding author.

Conflicts of Interest: The authors declare no conflict of interest.

Sample Availability: Samples of the compounds are not available from the authors.

\section{References}

1. Liu, Y.; Guo, J.; Zhu, E.; Liao, L.; Lee, S.-J; Ding, M.; Shakir, I.; Gambin, V.; Huang, Y.; Duan, X. Approaching the Schottky-Mott limit in van der Waals metal-semiconductor junctions. Nature 2018, 557, 696-700. [CrossRef]

2. Wang, Y.; Kim, J.C.; Wu, R.J.; Martinez, J.; Song, X.; Yang, J.; Zhao, F.; Mkhoyan, A.; Jeong, H.Y.; Chhowalla, M. Van der Waals contacts between three-dimensional metals and two-dimensional semiconductors. Nature 2019, 568, 70-74. [CrossRef]

3. Kim, B.-K.; Kim, T.-H.; Choi, D.-H.; Kim, H.; Watanabe, K.; Taniguchi, T.; Rho, H.; Kim, J.-J.; Kim, Y.-H.; Bae, M.-H. Origins of genuine Ohmic van der Waals contact between indium and $\mathrm{MoS}_{2}$. NPJ 2D Mater. Appl. 2021, 5, 9. [CrossRef]

4. $\quad$ Lau, C.S.; Chee, J.Y.; Ang, Y.S.; Tong, S.W.; Cao, L.; Ooi, Z.-E.; Wang, T.; Ang, L.K.; Wang, Y.; Chhowalla, M.; et al. Quantum transport in two-dimensional $\mathrm{WS}_{2}$ with high-efficiency carrier injection through indium alloy contacts. ACS Nano 2020, 14, 13700-13708. [CrossRef] 
5. Zhao, Q.; Jie, W.; Wang, T.; Castellanos-Gomez, A.; Frisenda, R. InSe Schottky diodes based on van der Waals contacts. Adv. Funct. Mater. 2020, 30, 2001307. [CrossRef]

6. Choi, D.-H.; Jang, S.; Jeong, D.-W.; Lee, J.-O.; Chang, H.; Ha, D.-H.; Lee, S.M.; Kim, J.; Suh, Y.D.; Bae, M.-H.; et al. Van-der-Waalsgap tunneling spectroscopy for single-wall carbon nanotubes. Carbon 2017, 113, 237-242. [CrossRef]

7. Murakami, T.; Matsumoto, K.; Kisoda, K.; Naito, R.; Nishio, K.; Isshiki, T.; Harima, H. Studies on the growth of pure double-walled carbon nanotube and its phonon spectra. J. Appl. Phys. 2008, 103, 114305. [CrossRef]

8. Appenzeller, J.; Knoch, J.; Radosavljević, M.; Avouris, P. Multimode transport in Schottky-barrier carbon-nanotube field-effect transistors. Phys. Rev. Lett. 2004, 92, 226802. [CrossRef] [PubMed]

9. Moon, S.; Song, W.; Lee, J.S.; Kim, N.; Kim, J.; Lee, S.-G.; Choi, M.-S. Eightfold shell filling in a double-wall carbon nanotube quantum dot. Phys. Rev. Lett. 2007, 99, 176804. [CrossRef] [PubMed]

10. Giusca, C.E.; Tison, Y.; Silva, S.R.P. Inter-layer interaction in double-walled carbon nanotubes evidenced by scanning tunneling microscopy and spectroscopy. Nano 2008, 3, 65-73. [CrossRef]

11. Soto, M.; Boyer, T.A.; Biradar, S.; Ge, L.; Vajtai, R.; Elías-Zúñiga, A.; Ajayan, P.M.; Barrera, E.V. Effect of interwall interaction on the electronic structure of double-walled carbon nanotubes. Nanotechnology 2015, 26, 165201. [CrossRef]

12. Blase, X.; Benedict, L.X.; Shirley, E.L.; Louie, S.G. Hybridization effects and metallicity in small radius carbon nanotubes. Phys. Rev. Lett. 1994, 72, 1878-1881. [CrossRef]

13. Qin, C.; Tian, Z.; Luo, X.; Xie, Q.; Nie, T.; Guo, X. First-principles study of electronic structure of double-walled and single-walled carbon nanotubes. Ceram. Int. 2021, 47, 2665-2671. [CrossRef]

14. Kim, B.-K.; Ahn, Y.-H.; Kim, J.-J.; Choi, M.-S.; Bae, M.-H.; Kang, K.; Lim, J.S.; López, R.; Kim, N. Transport measurement of Andreev bound states in a Kondo-correlated quantum dot. Phys. Rev. Lett. 2013, 110, 076803. [CrossRef] [PubMed]

15. Biercuk, M.J.; Mason, N.; Martin, J.; Yacoby, A.; Marcus, C.M. Anomalous conductance quantization in carbon nanotubes. Phys. Rev. Lett. 2005, 94, 026801. [CrossRef] [PubMed]

16. Delley, B. An all-electron numerical method for solving the local density functional for polyatomic molecules. J. Chem. Phys. 1990, 92, 508-517. [CrossRef]

17. Tkatchenko, A.; Scheffler, M. Accurate molecular van der Waals interactions from ground-state electron density and free-atom reference data. Phys. Rev. Lett. 2009, 102, 073005. [CrossRef]

18. Perdew, J.P.; Burke, K.; Ernzerhof, M. Generalized gradient approximation made simple. Phys. Rev. Lett. 1996, 77, 3865-3868. [CrossRef]

19. Wilder, J.W.G.; Venema, L.C.; Rinzler, A.G.; Smalley, R.E.; Dekker, C. Electronic structure of atomically resolved carbon nanotubes. Nature 1998, 391, 59-62. [CrossRef]

20. Odom, T.W.; Huang, J.-L.; Kim, P.; Lieber, C.M. Atomic structure and electronic properties of single-walled carbon nanotubes. Nature 1998, 391, 62-64. [CrossRef]

21. Zhao, S.; Yoo, S.; Wang, S.; Lyu, B.; Kahn, S.; Wu, F.; Zhao, Z.; Cui, D.; Zhao, W.; Yoon, Y.; et al. Tunneling spectroscopy in carbon nanotube-hexagonal boron nitride-carbon nanotube heterojunctions. Nano Lett. 2020, 20, 6712-6718. [CrossRef] [PubMed] 